\title{
The architecture of transcription elongation
}

A crystal structure explains how transcription factors enhance elongation and pausing

\section{By Thomas Fouqueau and Finn Werner}

The molecular machines that carry out transcription in all domains of lifebacteria, archaea, and eukaryotes - are multisubunit RNA polymerases (1). Over the past 15 years, structural analyses at ever higher resolution, in particular hybrid approaches that combine x-ray crystallography and single-particle cryo-electron microscopy, have provided detailed insights into how these enzymes work (2). On page 921 of this issue, Ehara et al. (3) apply such a multidisciplinary structural approach and in vitro transcription assays to reveal functional insights into a complete elongation complex from the yeast Komagataella pastoris, encompassing RNA polymerase II (RNAPII) and the transcription elongation factors Elf1, Spt4/5, and TFIIS. The results uncover the detailed molecular mechanisms by which these factors can not only enhance transcription elongation, but also pausing.

During transcription, RNAPII progresses repeatedly through the initiation, elongation, and termination phases of the transcription cycle (2). Distinct subsets of general transcription factors associate with RNAPII during each phase to form higherorder complexes that enable specific RNA polymerase functions. By inducing conformational changes in RNAPII, the transcription factors modulate its catalytic properties and its interactions with the template DNA and transcript RNA. Over the past decade, numerous studies have elucidated the structural basis of promoterbound transcription initiation complexes $(4,5)$. By contrast, structures of complete elongation complexes consisting of RNAPII and elongation factors have been sorely lacking. Ehara et al. now provide this missing link in our understanding of the architecture of transcription elongation.

RNAPII faces a substantial mechanical engineering challenge when proceeding from the initiation to the elongation phase of the transcription cycle. A highaffinity network of interactions between DNA-bound initiation factors and RNA polymerase is necessary for RNA polymerase recruitment to the gene promoter, but the same interactions need to be swiftly disrupted to enable the efficient release, or escape, of RNA polymerase into the gene body. 
Researchers have proposed that general elongation factors compete with initiation factors for RNA polymerase binding and that this swapping of factors enhances promoter escape $(6,7)$. Ehara et al.'s structure provides clues to the wider molecular basis of this phenomenon by revealing that the binding sites of initiation and elongation factors overlap. Furthermore, the binding of elongation factors enables an RNAPII architecture that is required for processive elongation and that can also alleviate or induce pausing in a regulated fashion.

RNAPII can carry out elongation unaided by factors, but when acting alone, is spontaneously and frequently interrupted by transcription pausing, which can result in a retrograde movement of RNAPII along the DNA (backtracking). Backtracked elongation complexes need to be reactivated by transcript cleavage factors such as TFIIS that penetrate deep into the RNAPII active center through the substrate entry pore (8). Ehara et al. reveal that an additional modus operandi of enhancing elongation prevails in the complex: Elongation factors Spt4/5 and Elf1 interact with RNAPII to form tunnel structures around the DNA and RNA (see the figure).

Spt4/5 is a general elongation factor that is conserved in all domains of life. In metazoans, it modulates transcription by regulating the pausing of RNAPII a short distance from the promoter. This process, called promoter proximal pausing, involves a negative elongation factor (NELF), but its structural basis is unknown (9). The elongation complex structure (3) provides unique insights into the function of Spt4/5, which consists of several distinct domains, including one NGN (NusG N-terminal) domain and five KOW (Kyrpides, Ouzounis, and Woese) domains. Ehara et al. show that the fifth KOW domain is necessary, and even sufficient, to stimulate elongation by RNAPII. This domain fits snugly into a concave depression of the RNAPII surface and thereby restricts conformational changes that may be counterproductive to elongation. In addition, several of the KOW domains shape both DNA-exit and RNAexit tunnels (see the figure). The NGN domain forms a lid on the DNA-binding channel of RNAPII, thereby preventing the dissociation of elongation complexes $(10,11)$.

The third factor included in Ehara et al.'s study, Elf1, is associated with elongating RNAPII throughout the genome (12). Elf1 binds close to the NGN domain and thereby seals the RNAPII DNA-entry tunnel entirely (see the figure). Ehara et al. show that Elf1 exerts an inhibitory effect on elongation, which hints at a possible role of Elf1 in regulating transcription. 
The complete elongation complex structure explains why mutations in Elf1, TFIIS, and Spt4/5 enhance each other, a phenomenon called synthetic lethality, which often implies that components interact physically, functionally, or both (13).

The eukaryotic and archaeal transcription machineries are closely related (2). Archaea use a smaller subset of factors than eukaryotes, but these correspond typically to a minimal configuration of factors that enable the most fundamental RNA polymerase functions during the transcription cycle. The conserved factors include all three elongation factors described in Ehara et al.'s study. Therefore, the structure of the complete elongation complex reflects the topology of the core transcription elongation machinery that lies at the heart of RNA synthesis in eukaryotes and archaea.

Important questions remain. For example, it is still unclear how the DNA tunnels affect elongation, whether the factors functionally cooperate during elongation, how the elongation process is regulated, and how NELF interacts with Spt4/5. Armed with Ehara et al.'s transformative structural information, biochemists can now test working hypotheses predicted from the structure to pave the way for a thorough understanding of the mechanisms of transcription elongation and pausing. 


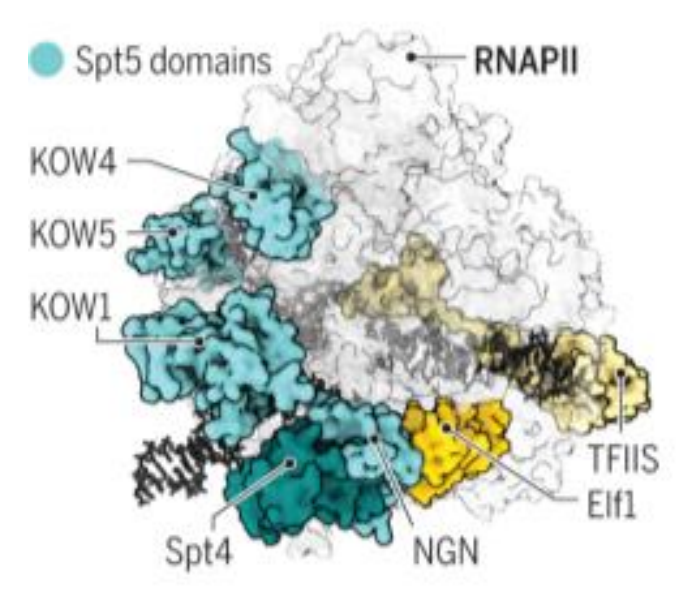

\section{Tunnels for transcription}

The general transcription elongation factors Spt4/5, Elf1. and TFIIS bind to RNAPII and form a streamlined molecular machine where the DNA template and RNA transcript are encased in tunnel-like structures.
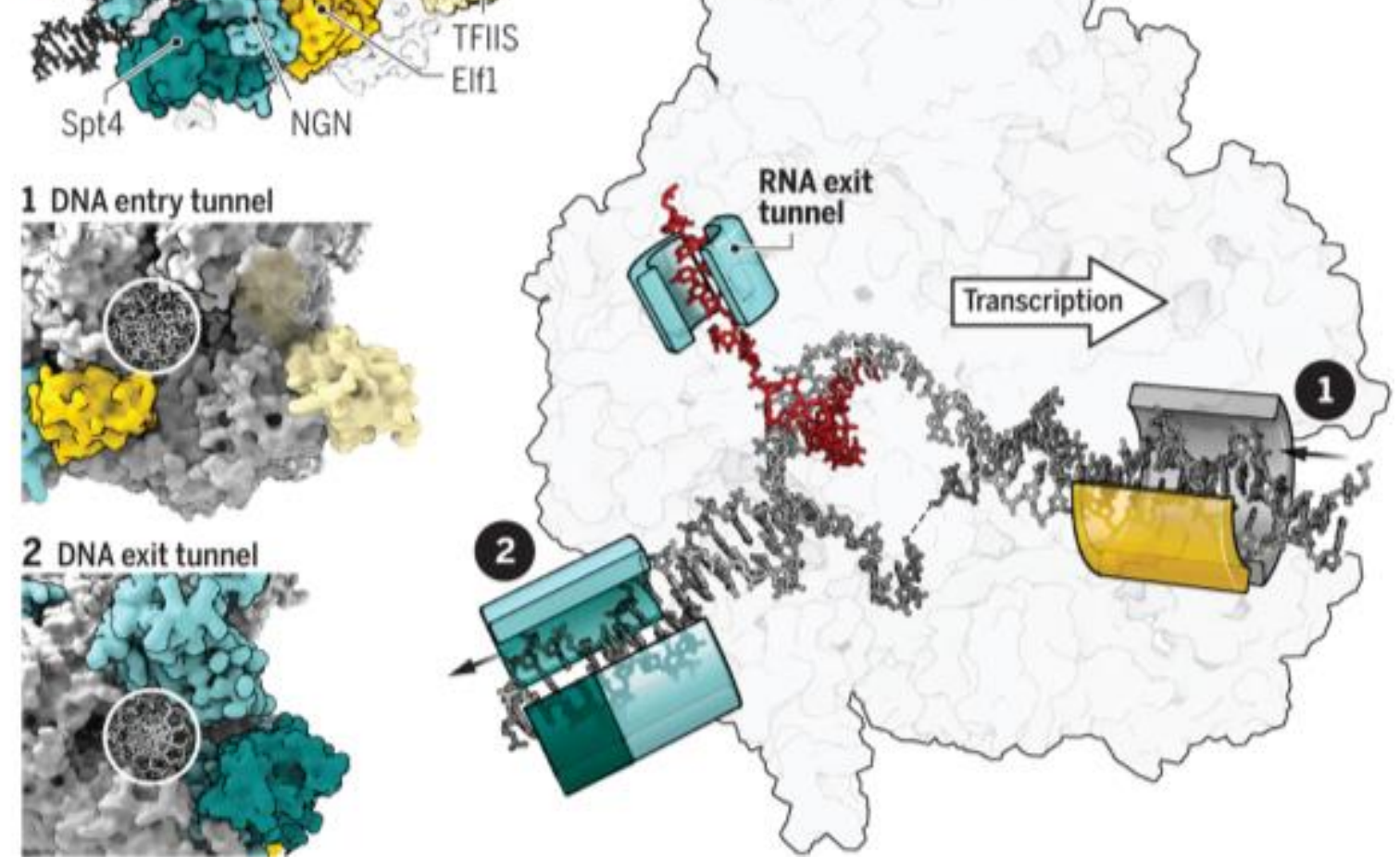

\section{Figure: Tunnels for transcription}

The general transcription elongation factors Spt4/5, Elf1, and TFIIS bind to RNAPII and form a streamlined molecular machine where the DNA template and RNA transcript are encased in tunnel-like structures. 


\section{REFERENCES}

1. F. Werner, D. Grohmann, Nat. Rev. Microbiol. 9, 85 (2011).

2. D. Elmlund, S. N. Le, H. Elmlund, Curr. Opin. Struct. Biol. 46, 1 (2017).

3. H. Ehara et al., Science 357, 921 (2017).

4. Y. He, J. Fang, D. J. Taatjes, E. Nogales, Nature 495, 481 (2013).

5. C. Plaschka et al., Nature 533, 353 (2016).

6. D. Grohmann et al., Mol. Cell 43, 263 (2011).

7. S. Schulz et al., Proc. Natl. Acad. Sci. U.S.A. 113, E1816 (2016).

8. A. C. Cheung, P. Cramer, Nature 471, 249 (2011).

9. K. Adelman, J. T. Lis, Genetics 13, 720 (2012).

10. A. Hirtreiter et al., Nucleic Acids Res. 38, 4040 (2010).

11. F. Werner, J. Mol. Biol. 417, 13 (2012).

12. A. Mayer et al., Nat. Struct. Mol. Biol. 17, 1272 (2010).

13. D. Prather, N. J. Krogan, A. Emili, J. F. 